\title{
Decomposition of cartesian products of regular graphs into isomorphic trees*
}

\author{
Kyle F. Jao, Alexandr V. Kostochka and Douglas B. West \\ Dedicated to the memory of Hunter Snevily
}

\begin{abstract}
We extend the ideas of Snevily and Avgustinovitch to enlarge the families of $2 m$-regular graphs and $m$-regular bipartite graphs that are known to decompose into isomorphic copies of a tree $T$ with $m$ edges. For example, consider $r_{1}, \ldots, r_{k}$ with $\sum_{i=1}^{k} r_{i}=m$. If $T$ has a $k$-edge-coloring with $r_{i}$ edges of color $i$ such that every path in $T$ uses some color once or twice, then every cartesian product of graphs $G_{1}, \ldots, G_{k}$ such that $G_{i}$ is $2 r_{i}$-regular for $1 \leq i \leq k$ decomposes into copies of $T$.
\end{abstract}

AMS 2000 SUbJeCt ClASSifications: Primary 05C51, 05 C01.

KEYWORDS AND PHRASES: Isomorphic decomposition, cartesian product, regular graph, tree, edge-coloring.

\section{Introduction}

Ringel [8] conjectured that for every tree $T$ with $m$ edges, the complete graph $K_{2 m+1}$ decomposes into copies of $T$, meaning that the edges of $K_{2 m+1}$ can be partitioned into classes forming copies of $T$. Such a partition is a $T$-decomposition. Häggkvist [3] conjectured more generally that every $2 m$ regular graph has a $T$-decomposition. Graham and Häggkvist [3] conjectured that every $m$-regular bipartite graph has a $T$-decomposition. The restriction to bipartite graphs for $T$-decomposition of $m$-regular graphs is due to the elementary observation that an $m$-regular graph decomposes into copies of $K_{1, m}$ if and only if it is bipartite.

In this paper we broaden the classes of instances where the conjectures of [3] are known to hold. We begin by reviewing earlier results.

Theorem 1.1 (Snevily [5]). Let $T$ be a tree with $m$ edges. If $G$ is $2 m$-regular and has girth greater than the diameter of $T$, then $G$ has a $T$-decomposition.

*This work was supported in part by the NSF under Award No. DMS0099608 (A. V. Kostochka) and by the NSA under Award No. H98230-10-1-0363 (D. B. West). 
Häggkvist [3] stated without proof the stronger result that girth at least $\operatorname{diam} T$ suffices.

Theorem 1.2 (Snevily [5]). If $T$ is a tree with $m$ edges, and $G$ is the cartesian product of $m$ cycles, then $G$ has a $T$-decomposition.

Theorem 1.3 (Snevily [5]). If $T$ is a tree with $m$ edges, and $G$ is the cartesian product of a $2 l$-cycle and $m-2$ copies of $K_{2}$, then $G$ has a $T$ decomposition.

The special case of Theorem 1.3 with $l=2$ and $m \geq 2$ is the $m$ dimensional hypercube; this case was solved earlier by Fink [2]. Fink also showed that the trees in the decomposition could be required to be induced subgraphs. Our main result is Theorem 2.1, giving more general sufficient conditions for $T$-decompositions. It yields the various corollaries listed below, which strengthen the results above.

Corollary 1.4. Let $T$ be an edge-colored tree such that every path $P$ in $T$ uses some color that appears on at most $q$ edges of $P$. If the color classes have sizes $r_{1}, \ldots, r_{k}$, and $G$ is the cartesian product of regular graphs of degrees $2 r_{1}, \ldots, 2 r_{k}$, each having girth greater than $q$, then $G$ has a $T$-decomposition.

For $r_{1}=\cdots=r_{k}=1$, Corollary 1.4 yields Theorem 1.2. For $k=1$ and general $r_{1}=m$, it becomes Theorem 1.1 . In this case $(k=1$ and no cartesian products), there has been some work on decompositions into special trees.

Theorem 1.5 (Kouider and Lonc [6]). For $m \leq 2 g-3$, every $2 m$-regular graph with girth at least $g$ decomposes into paths of length $\mathrm{m}$.

Theorem 1.5 strengthens Theorem 1.1 for the special case of paths. We will use the case $m=3$ of their technically stronger version of Theorem 1.5 in giving an application of our theorem. Meanwhile, for $2 m$-regular graphs our Theorem 2.1 says the following, which essentially is implicit in Snevily's proof of Theorem 1.1. (Neither of Corollary 1.6 and Theorem 1.5 implies the other.)

Corollary 1.6. Let $T$ be a tree with $m$ edges, and let $G$ be a $2 m$-regular graph. If $G$ has a 2-factorization such that every cycle consisting of edges from distinct 2-factors has length greater than the diameter of $T$, then $G$ has a T-decomposition.

Finally, for cartesian products of bipartite graphs our theorem yields the following, which becomes Theorem 1.3 when $r_{1}=2$ and $r_{2}=\cdots=r_{k}=1$ and the factors are connected. 
Corollary 1.7. Let $T$ be an edge-colored tree such that every path $P$ in $T$ uses some color that appears on at most three edges of $P$. If the color classes have sizes $r_{1}, \ldots, r_{k}$, and $G$ is the cartesian product of regular bipartite graphs of degrees $r_{1}, \ldots, r_{k}$, then $G$ has a $T$-decomposition.

Snevily [5] proved his results by seeking more structure in the decompositions. He labeled $V(T)$ and required each vertex of $G$ to appear with distinct labels in the copies of $T$ incident to it. Avgustinovich [1] obtained results on decompositions of bipartite graphs into induced copies of $T$ by considering labels on the edges of $T$. We combine and extend these ideas to give a general sufficient condition in Theorem 2.1 for the existence of a $T$-decomposition of $G$ when $G$ is a $2 m$-regular cartesian product of regular graphs with even degree. (The theorem also includes an analogous result for $m$-regular cartesian products of regular bipartite graphs.)

We employ Avgustinovich's edge-labeling idea in the sense of coloring the edges of $T$. When $G$ is the cartesian product of $G_{1}, \ldots, G_{k}$ and $G_{i}$ is $2 r_{i^{-}}$ regular, with $\sum r_{i}=m$, we give color $i$ to $r_{i}$ edges in $T$. The existence of a suitable edge-coloring guarantees the decomposition. As in Snevily's results, we guarantee a decomposition having a stronger property to facilitate the inductive proof. Each vertex appears in $m+1$ copies of $T$, once representing each of the $m+1$ vertices in a numbering of $V(T)$.

As suggested in Corollary 1.6, our general sufficient condition in Theorem 2.1 permits more delicate interaction between the edge-coloring of $T$ and chosen 2-factorizations of $G_{1}, \ldots, G_{k}$, rather than just imposing girth requirements on $G_{1}, \ldots, G_{k}$. Girth requirements are one way to ensure that the hypotheses of Theorem 2.1 hold. In Sections 3-5, we study conditions on $r$ to guarantee that $T$ has an edge-coloring of the type needed to guarantee (via Theorem 2.1) that a $T$-decomposition will exist regardless of the girth or choice of 2-factorizations in $G_{1}, \ldots, G_{k}$. To make this precise, we introduce some terminology.

Definition 1.8. Throughout this paper, let $r=\left(r_{1}, \ldots, r_{k}\right)$. Given a $k$ tuple $r$ with sum $m$, an edge-coloring of a tree with $m$ edges is $r$-exact if it has $r_{i}$ edges of color $i$, for $1 \leq i \leq k$. We always index the multiplicities so that $r_{1} \leq \cdots \leq r_{k}$. An edge-coloring of a tree $T$ is $q$-good if every path in $T$ has some color appearing on it that appears at most $q$ times on it (such a path is $q$-bounded).

Corollary 1.4 states that if $T$ has a 2-good $r$-exact edge-coloring, then every product of simple regular graphs with degrees $2 r_{1}, \ldots, 2 r_{k}$ has a $T$ decomposition. (Similarly, when each $G_{i}$ is bipartite and $r_{i}$-regular, one seeks a 3-good $r$-exact edge-coloring, since the product has girth at least 4.) 
When $r_{1} \geq 3$ (and hence $m / k \geq 3$ ), the path $P_{m}$ has no 2 -good $r$ exact edge-coloring. Nevertheless, we will study circumstances with $m / k<4$ under which a coloring that guarantees $T$-decompositions exists.

A tree $T$ is special if it has a vertex $x$ such that every component of $T-x$ has at most two edges. Large special trees are very far from paths. In Section 3, we discuss when special trees have 2 -good $r$-exact edge-colorings.

In Section 4 we introduce a weaker restriction on edge-colorings. An edge-coloring of $T$ is weakly 2-good if every path in $T$ is either 2-bounded or has a color appearing only on a 3-edge subpath whose two internal vertices have degree 2 in $T$. Using a result of Kouider and Lonc [6] on decomposition of regular graphs, we apply our general condition in Theorem 2.1 to prove that if $T$ has a weakly 2-good $r$-exact edge-coloring, then again every cartesian product of regular graphs with degrees $2 r_{1}, \ldots, 2 r_{k}$ has a $T$-decomposition.

By using the results on 2-good edge-colorings of special trees, we show that $m / k<4$ and $r_{k} \leq\left\lceil\frac{m+1}{2}\right\rceil$ together guarantee weakly 2-good $r$-exact edge-colorings of all trees with $m$ edges. Certain cases in our inductive proof of this result require splitting the list $r$ into two lists with sum $r$ to which the induction hypothesis can be applied. In particular, one needs each list in the split to have sufficiently many nonzero terms. The splittability results are of interest on their own. They are the most difficult technical results of the paper, so we postpone their proofs to Section 5.

\section{The general decomposition theorem}

Let $G$ be the cartesian product of regular graphs $G_{1}, \ldots, G_{k}$. The product decomposes naturally into copies of $G_{1}, \ldots, G_{k}$, which yields a natural $k$ coloring of $E(G)$ by giving color $i$ to the edges whose endpoints differ in the $i$ th coordinate (this coordinate coloring forms copies of $G_{i}$ ). To produce a $T$ decomposition of $G$, we similarly color $E(T)$ with $k$ colors, and the inductive proof will produce a decomposition in which, for each $i$, the edges of color $i$ in each copy of $T$ belong to copies of $G_{i}$ in the coordinate coloring of $G$. Thus the sizes $r_{1}, \ldots, r_{k}$ of the color classes in $T$ must be proportional to the sizes of $G_{1}, \ldots, G_{k}$.

We require further structure for the coloring and the decomposition. Our approach works in two settings: either each $G_{i}$ is a $2 r_{i}$-regular graph, or each $G_{i}$ is an $r_{i}$-regular bipartite graph. In each case, we use a factorization $\mathbf{F}_{i}$ of each $G_{i}$. In the nonbipartite case, $\mathbf{F}_{i}$ is a 2-factorization, guaranteed to exist by Petersen's Theorem [7]. In the bipartite case, $\mathbf{F}_{i}$ is a 1-factorization, guaranteed to exist by the Marriage Theorem of Frobenius and König [4]. 
In both cases, $\mathbf{F}_{i}$ consists of $r_{i}$ factors. Given a one-to-one correspondence between $\mathbf{F}_{i}$ and the set of edges with color $i$ in $T$, our $T$-decomposition of $G$ embeds each edge of $T$ with color $i$ along an edge arising from the corresponding factor in $\mathbf{F}_{i}$.

Theorem 2.1. Let $T$ be a tree with $m$ edges. Let $r$ be a nondecreasing $k$ tuple with sum $m$. Color $E(T)$ so that $r_{i}$ edges have color $i$. Let $G$ be the cartesian product of multigraphs $G_{1}, \ldots, G_{k}$, where

Case 1: each $G_{i}$ is an $r_{i}$-regular bipartite multigraph, or

Case 2: each $G_{i}$ is a $2 r_{i}$-regular multigraph.

Consider Case $j$, where $j \in\{1,2\}$. For $1 \leq i \leq k$, let $\mathbf{F}_{i}$ be a $j$-factorization of $G_{i}$, and establish a one-to-one correspondence that pairs each edge of color $i$ in $T$ with one factor in $\mathbf{F}_{i}$. If every path $P$ in $T$ has an edge of some color $h$ such that $G_{h}$ has no cycle with edges in distinct $\mathbf{F}_{h}$-classes all corresponding to edges of $P$, then $G$ has a $T$-decomposition.

Proof. The proofs for both Cases are very similar, so we combine most of the discussion by considering Case $j$. As described above, the coordinate coloring gives color $i$ to each edge of $G$ whose endpoints differ in coordinate $i$ in the cartesian product. Furthermore, the $j$-factorizations $\mathbf{F}_{1}, \ldots, \mathbf{F}_{k}$ yield a canonical $j$-factorization of $G$ by decomposing each copy of $G_{i}$ according to $\mathbf{F}_{i}$ and combining these decompositions. Thus each edge of $T$ corresponds to a $j$-factor of $G$.

We prove a stronger result by induction on $m$. We produce a $T$-decomposition such that in each copy of $T$, each edge $e$ is embedded as an edge of the $j$-factor in $G$ corresponding to $e$. Furthermore, each vertex of $G$ represents distinct vertices of $T$ in the copies of $T$ using it in the decomposition. More precisely, in Case 2 each vertex of $G$ appears in $m+1$ copies of $T$, once as each vertex of $T$. In Case 1 , with $T$ having partite sets $X^{\prime}$ and $Y^{\prime}$, and $G$ having partite sets $X$ and $Y$, each vertex of $X$ appears in $\left|X^{\prime}\right|$ copies of $T$, once as each vertex of $X^{\prime}$, and similarly for $Y$ and $Y^{\prime}$.

For $m=1$, the claim is immediate. In Case 1, $G$ consists of isolated edges that can be labeled as desired. In Case 2, follow the cycles in the single 2-factor, labeling each edge in order with the two leaves of $T$.

For $m>1$, let $u$ be a leaf of $T$, with neighbor $v$, and let $T^{\prime}=T-u$. By symmetry, we may assume that $u v$ has color $k$ in the coloring of $E(T)$. Let $H$ be the $j$-factor of $G_{k}$ in $\mathbf{F}_{k}$ that corresponds to $u v$.

Let $G^{\prime}$ be the graph obtained by deleting $E(H)$ from all copies of $G_{k}$ in the product. Thus $G^{\prime}$ is the cartesian product of $G_{k}-E(H)$ with all of $G_{1}, \ldots, G_{k-1}$ (when $k=1$, this degenerates to $G=G_{k}$ and $G^{\prime}=G-$ 
$E(H))$. Since the paths in $T^{\prime}$ are contained in $T$, deleting $E(H)$ leaves $j$ factorizations that satisfy the hypotheses for $G^{\prime}$. Consider the $T^{\prime}$-decomposition of $G^{\prime}$ provided by applying the induction hypothesis to $G^{\prime}$.

In Case 1, we may assume by symmetry that $v \in X^{\prime}$, and for each $w \in X$ we let $w y$ be the edge incident to $w$ in $H$. In Case 2, for each $w \in V(G)$ we let $y$ be the vertex following $w$ on the cycle through $w$ in $H$ (along a consistent orientation of the cycle).

We extend the copy $\hat{T}$ of $T^{\prime}$ having $v$ at $w$ by adding the edge $w y$. To see that $y$ is not already in $\hat{T}$, suppose that it is, and let $P$ be the path from $w$ to $y$ in $\hat{T}$. The edges of a single color $i$ along $P$ correspond to distinct $j$-factors in $\mathbf{F}_{i}$. The edge $w y$ in color $k$ corresponds to a different $j$-factor in $\mathbf{F}_{k}$ from the others in color $k$ along $P$. Together, $P$ and $w y$ complete a cycle $C$ in $G$. If color $i$ appears on $C$, then $C$ collapses to a nontrivial closed trail in $G_{i}$ using edges from different $j$-factors in $\mathbf{F}_{i}$. This closed trail contains a cycle in $G_{i}$ through distinct $j$-factors. This statement holds for every color that appears on $P$, which contradicts the hypothesis about paths in $T$.

Hence $y \notin V(\hat{T})$, and the extensions are copies of $T$. Furthermore, the required stronger statements about the placement of edges and vertices in the decomposition are preserved.

There is no obvious common generalization of Cases 1 and 2 .

Example 2.2. If $G$ is the cartesian product of a $2 r$-regular graph $C$ and an $s$-regular bipartite graph $B$, one would seek a $T$-decomposition of $G$, where $T$ has $r+s$ edges. When $C=K_{3}$ and $B=K_{3,3}$, we have $r=1$ and $s=3$, but the product has 45 edges, and 45 is not divisible by 4 .

In the rest of this section, we study paths. We begin with a simple way to guarantee $q$-good edge-colorings.

Definition 2.3. A $k$-tuple $r$ is greedily $q$-good if $r_{i} \leq q\left(1+\sum_{j<i} r_{j}\right)$ for all $i$.

Corollary 2.4. Let $T$ be a path with $m$ edges, and let $r$ be a $k$-tuple of positive integers with sum $m$. Let $G$ be the cartesian product of graphs $G_{1}, \ldots, G_{k}$. If each $G_{i}$ is $2 r_{i}$-regular and $r$ is greedily 2 -good, then $G$ has a $T$-decomposition. If each $G_{i}$ is bipartite and $r$ is greedily 3-good, then $G$ has a T-decomposition.

Proof. Since a vacuous sum is 0 , we have $r_{1} \leq 2$ in the first case and $r_{1} \leq 3$ in the second.

Consider the first statement. By Corollary 1.4, it suffices to partition $E(T)$ into color classes of sizes $r_{1}, \ldots, r_{k}$ such that each subpath uses a color that appears at most twice on it, since each $G_{i}$ has girth at least 3. 
Starting with $r_{1}$ copies of 1 , we inductively produce a list of colors in order for the edges. To add copies of $i$, insert at most two copies of $i$ in each space between entries of the previous list. Since $r_{i} \leq 2\left(1+\sum_{j=1}^{i-1} r_{j}\right)$, there is enough room to do this.

To complete the proof, observe that on every subpath, the smallest label appears at most twice. This holds because a path with three copies of $i$ on it must have an smaller label on some internal edge.

Since bipartite graphs have girth at least 4, the analogous argument works for the second statement, using Corollary 1.7.

Lemma 2.5. If $m / k<q+1$, then $r$ is greedily q-good, and hence $P_{m}$ has a q-good r-exact edge-coloring.

Proof. If $\sum_{j \leq i} r_{j} \geq i(q+1)$ for some $i$, then $r_{j} \geq q+1$ for $j \geq i$, since $r$ is nondecreasing. Hence $m \geq \sum_{j=1}^{k} r_{j} \geq k(q+1)$, contradicting $m<k(q+1)$. Therefore, we have $\sum_{j \leq i} r_{j}<i(q+1)$ for each $i$. Also, $i-1 \leq \sum_{j<i} r_{j}$, so

$$
r_{i}+\sum_{j<i} r_{j}<i(q+1) \leq q+1+(q+1) \sum_{j<i} r_{j}
$$

which simplifies to $r_{i} \leq q\left(1+\sum_{j<i} r_{j}\right)$.

Being greedily 2-good is not a necessary condition for $P_{m}$ to have a 2good $r$-exact edge-coloring. For example, when $r=(2,26,26,26)$, still there is a 2-good $r$-exact edge-coloring of $P_{81}$. On the other hand, Lemma 2.5 is sharp: some lists satisfying $m / k<q+1$ are not greedily $(q-1)$-good, and the ratio $m / k$ needed to guarantee $q$-good $r$-exact colorings for general trees must be much smaller.

Example 2.6. Define $r$ by $r_{i}=1$ for $1 \leq i \leq k-1$ and $r_{k}=q k$. Since $\sum r_{i}=(q+1) k-1$, the ratio condition holds, but $r_{k}=q k>(q-1) k=$ $(q-1)\left(1+\sum_{i<k} r_{i}\right)$. Hence $r$ is not greedily $(q-1)$-good.

Similarly, if $r_{1}=q$ and $r_{i}=q+1$ for $2 \leq i \leq k$, then $\sum r_{i}=(q+1) k-1$, but $r_{1}>q-1$. Again $r$ is not greedily $(q-1)$-good.

Now consider a tree $T$ having one central vertex of degree $k+1$ that is a common endpoint of $k+1$ paths of length $\lceil(q+1) / 2\rceil$. Thus $m=$ $(k+1)\lceil(q+1) / 2\rceil$, so $m / k$ is just over half of $q+1$. Let $r_{i}=1$ for $i<k$ and $r_{k}=m-k+1$. Every $r$-exact edge-coloring leaves two branches completely in color $k$, forming a monochromatic path of length at least $q+1$.

Example 2.6 suggests that general trees are much more difficult to handle than paths. 


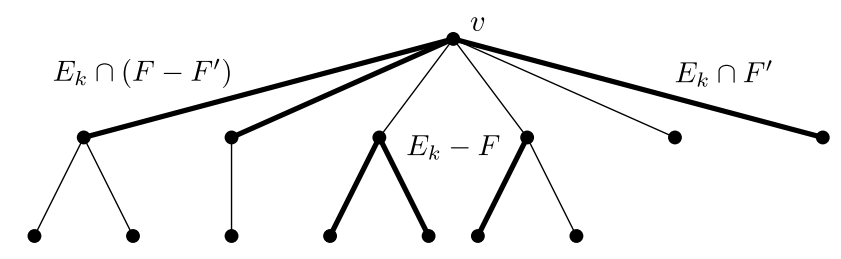

Figure 1: The set $E_{k}$ for Proposition 3.1.

\section{2-good edge-colorings of special trees}

We now restrict our attention to Case 2: $G$ is the cartesian product of $G_{1}, \ldots, G_{k}$, where each $G_{i}$ is $2 r_{i}$-regular. Let $r=\left(r_{1}, \ldots, r_{k}\right)$, indexed in nondecreasing order, and let $m=\sum r_{i}$. If the factors are simple graphs, then every cycle contains at least three edges. In this case, if $T$ has a 2 -good $r$ exact edge-coloring, then Theorem 2.1 implies that $G$ has a $T$-decomposition. Thus it is natural to ask (1) when does a tree have such an edge-coloring, and (2) are there weaker conditions than 2-good edge-coloring for $T$ that guarantee a $T$-decomposition of $G$ ?

For simplicity, we always assume that $T$ has $m$ edges and $r$ is a nondecreasing list of $k$ positive integers with sum $m$. Let $\ell(v)$ be the number of leaf neighbors of a vertex $v$ in $T$.

Proposition 3.1. If $T$ has a 2-good r-exact edge-coloring, then $r_{k} \leq m-$ $d(v)+\max \{\ell(v), 1\}$ for all $v \in V(T)$. In addition, $r_{k}>m-d(v)+\ell(v)$ only when $\ell(v)=0$ and the components of $T-v$ are all stars.

Proof. Given a 2-good $r$-exact edge-coloring of $T$, let $E_{k}$ be the set of edges having color $k$. Fix $v \in V(T)$. Let $F$ be the set of edges incident to $v$ and $F^{\prime}$ be the subset of $F$ consisting of edges incident to leaves of $T$. If $\left|E_{k} \cap F\right| \leq 1$, then $r_{k} \leq m-d(v)+1$, since otherwise there is a path of length 3 in color $k$. If $\left|E_{k} \cap F\right| \geq 2$, then the edges in $E_{k}-F$ are not incident to any edge in $E_{k} \cap F$. Each edge of $E_{k} \cap\left(F-F^{\prime}\right)$ is incident to at least one edge that is not incident to $v$ and does not lie in $E_{k}$ (see Fig. 1). Thus $\left|E_{k}-F^{\prime}\right|=\left|E_{k}-F\right|+\left|E_{k} \cap\left(F-F^{\prime}\right)\right| \leq m-d(v)$. Since $\left|E_{k} \cap F^{\prime}\right| \leq \ell(v)$, we have $\left|E_{k}\right|=\left|E_{k}-F^{\prime}\right|+\left|E_{k} \cap F^{\prime}\right| \leq m-d(v)+\ell(v)$.

If $r_{k}>m-d(v)+\ell(v)$, then $\ell(v)=0$ and $r_{k}=m-d(v)+1$, which requires that $\left|E_{k} \cap F\right|=1$ and all edges not incident to $v$ have color $k$. Therefore, every component of $T-v$ has no 3 -edge path and is a star.

Proposition 3.1 suggests the question of when this condition is sufficient. Unfortunately, it is not sufficient even for trees with diameter 4. 
Example 3.2. Given $a \geq 3$ and $b \geq 2$, let $T_{a, b}$ be the tree having a vertex $x$ with $d(x)=a$ such that every component of $T-x$ is a star with $b$ edges whose center is adjacent to $x$. Consider $r=\left(r_{1}, r_{2}\right)$ with $a+b \leq r_{1} \leq m / 2$ and $r_{1}+r_{2}=m$. Note that $\ell(x)=0$ and $m=a+a b$. Note also that always $r_{2} \leq m-d(x)-b$, so the necessary condition holds.

We show that $T_{a, b}$ has 2-good $r$-exact edge-colorings only when $r_{1}-a$ is a multiple of $b-1$. Suppose $T_{a, b}$ has a 2 -good $r$-exact edge-coloring. Let $F$ be the set of edges incident to $x$, and let $i$ be the number of edges in $F$ having color 1.

Since $r_{2} \geq m / 2 \geq a+b$, we have $r_{2}>a$, and therefore $i \geq 1$. If $i=1$, then at least two edges in $F$ have color 2 , since $a \geq 3$. The pendant edges incident to an edge in $F$ of color 2 now must have color 1 ; otherwise we get a monochromatic path of length 3. Hence $r_{1} \geq 1+(a-1) b$. Since $r_{1} \leq m / 2$, we have $1+(a-1) b \leq(a+a b) / 2$, which simplifies to $(a-2)(b-1) \leq 0$, a contradiction. If $i \geq 2$, then the pendant edges incident to an edge of color 1 have color 2. If $i \geq a-1$, then $r_{1} \leq a-1+b$, which contradicts $r_{1} \geq a+b$. Therefore, at least two edges in $F$ have color 2 .

In the remaining case, $2 \leq i \leq a-2$. Now the color of every pendant edge differs from the color of the edge in $F$ incident to it. Hence $r_{1}=a-i+i b$. Consequently, if $a+b \leq r_{1} \leq m / 2$ and $r_{1}-a$ is not a multiple of $b-1$, then $T_{a, b}$ with $a \geq 3$ and $b \geq 2$ has no 2-good $r$-exact edge-coloring. For the degenerate case $b=1$, a 2-good $r$-exact edge-coloring exists only when $r_{1} \in\{a-1, a\}$.

Nevertheless, the condition is sufficient for a special family of trees with diameter 4 .

Definition 3.3. Given the nondecreasing list $r$ of length $k$, define a function $c_{r}:[m] \rightarrow[k]$ by letting $c_{r}(t)$ be the least index $h$ such that $t \leq \sum_{i \leq h} r_{i}$.

Lemma 3.4. Let $T$ be a tree consisting of paths of length at most 2 having a common endpoint $x$. If $r_{k} \leq m-d(v)+\max \{\ell(v), 1\}$ for all $v \in V(T)$, then $T$ has a 2-good r-exact edge-coloring.

Proof. Note that $m-d(v)+\max \{\ell(v), 1\}$ is minimized when $v=x$. Note also that $m=2 d(x)-\ell(x)$. Index the edges as $e_{1}, \ldots, e_{m}$ so that the first $d(x)-\ell(x)$ edges are the non-pendant edges incident to $x$, the next $\ell(x)$ edges are the pendant edges incident to $x$, and the last $m-d(x)$ edges are the edges not incident to $x$, with $e_{t}$ incident to $e_{t-d(x)}$ for $d(x)<t \leq m$. Let the color assigned to edge $e_{t}$ be $c_{r}(t)$. By construction, this coloring is $r$-exact; we claim that it also is 2-good.

Suppose that $P$ is a monochromatic 3-edge path in this coloring. Let $e_{t}$ be the edge in $P$ with least index. Note that $1 \leq t \leq d(x)-\max \{\ell(x), 1\}$. 
It follows that $e_{t^{\prime}}$ gets color $c_{r}(t)$ for all $t^{\prime}$ with $t \leq t^{\prime} \leq t+d(v)$, and hence $r_{c_{r}(t)} \geq d(x)+1$. If also $c_{r}(t)<k$, then $r_{c_{r}(t)}+r_{k}>2 d(x) \geq m$. Thus $c_{r}(t)=k$, so $e_{t^{\prime}}$ gets color $k$ for $t \leq t^{\prime} \leq m$. Therefore, $r_{k} \geq m-t+1 \geq$ $m-d(x)+\max \{\ell(x), 1\}+1$, a contradiction.

Example 3.2 shows that when $a \geq 3$ and $b \geq 2$, the condition $r_{k} \leq$ $m-d(v)+\max \{\ell(v), 1\}$ for all $v$ is not sufficient for $T_{a, b}$ to have a 2-good $r$-exact edge-coloring. Lemma 3.4 includes the degenerate case of $T_{a, b}$ when $b=1$. We next consider a generalization of $T_{a, 2}$. A special tree is a tree $T$ having a special vertex $x$ such that every component of $T-x$ has at most two edges. Although the condition on $r_{k}$ in Lemmas 3.4 is not sufficient to guarantee 2-good $r$-exact edge-colorings for special trees (as in Example 3.2 with $b=2$ ), we will prove in Lemma 3.7 that it does suffice for special trees when also $m / k<4$ and $m \geq 8$. We first prove a lemma about a special subclass of special trees.

Lemma 3.5. Let $T$ be a tree consisting of $d_{1}$ paths of length $1, d_{2}$ paths of length 2 , and $d_{3}$ paths of length 3 having a common endpoint $x$. Let $j=c_{r}\left(d_{2}+d_{3}\right)$. If $r_{j} \leq d(x)+\sum_{i<j} r_{i}$ when $c_{r}\left(d_{3}\right)=j$, or $r_{j} \leq m-d(x)+\ell(x)$ when $c_{r}\left(d_{3}\right)<j$, then $T$ has a 2-good r-exact edge-coloring such that on each path with endpoint $x$ the edge incident to $x$ gets a color distinct from the colors assigned to the other edges of that path.

Proof. Consider the multiset $U$ consisting of $r_{i}$ copies of color $i$ for $1 \leq i \leq k$; note that $U$ has size $d_{1}+2 d_{2}+3 d_{3}$. Let $S$ be a multiset consisting of $d_{2}+d_{3}$ smallest elements of $U$ (since $j=c_{r}\left(d_{2}+d_{3}\right)$, they are all at most $j$ ), and let $R=U-S$. We will partition $U$ into multisets assigned to the components of $T-x$ (we just call them "sets"). A component of $T-x$ having $p$ vertices gets a set of size $p$ to be used on its edges and the edge joining it to $x$. We form the sets of size 1 , then size 3 , then size 2 . First let $d_{1}$ smallest elements of $R$ be the sets of size 1 . Next iteratively associate a smallest remaining element of $S$ with two smallest remaining elements of $R$; do this $d_{3}$ times. Finally, associate a smallest remaining element of $S$ with a smallest remaining element of $R$.

This procedure creates the desired sets if in each set the smallest element occurs only once, which holds by construction when the smallest element is less than $j$. Since the smallest element in sets of size at least 2 comes from $S$ and is always at most $j$, it suffices to show that when the smallest element is $j$ there is no other $j$ in the set. We bound the multiplicity of $j$ in two cases.

Case 1: $c_{r}\left(d_{3}\right)=j$. In this case, at most $d_{1}$ copies of $j$ form sets of size 1 . In the step forming sets of size 3 , at most two copies of $j$ remaining in $R$ are associated with each element of $S$ that is less than $j$ (there are 
$\sum_{i<j} r_{i}$ of them). We need that at most one copy of $j$ in $S$ (there are $|S|-\sum_{i<j} r_{i}$ of them) and no copy of $j$ in $R$ appears in each set of size 2 and in each other set of size 3 . Hence it is necessary and sufficient to have $r_{j} \leq d_{1}+2 \sum_{i<j} r_{i}+|S|-\sum_{i<j} r_{i}$. This is equivalent to the hypothesis, since $|S|=d_{2}+d_{3}$ and $d(x)=d_{1}+d_{2}+d_{3}$.

Case 2: $c_{r}\left(d_{3}\right)<j$. In this case, we need that at most two copies of $j$ in $R$ appear in each set of size 3 formed, and at most one copy of $j$ appears in each set of size 2 . Hence it is necessary and sufficient to have $r_{j} \leq d_{1}+2 d_{3}+d_{2}$. Since $\ell(x)=d_{1}$ and $m=d_{1}+2 d_{2}+3 d_{3}$, the required inequality is equivalent to that given in the hypothesis.

Remark 3.6. In Corollary 2.4, the condition $r_{i} \leq 2\left(1+\sum_{j<i} r_{i}\right.$ ) (for all $i$ ) is shown to be sufficient for a path to have a 2-good $r$-exact edge-coloring. Lemma 2.5 shows that if $m / k<3$, then that condition always holds, and hence a 2-good $r$-exact edge-coloring of the path exists. However, when $m / k<3$ is changed to $m / k<4$, the full path is not 2-bounded when $m \geq 6$ and $r_{i}=3$ for all $i$.

For special trees with $m \geq 8$, the condition $m / k<4$ suffices as long as $r_{k}$ is not too big.

Lemma 3.7. Let $T$ be a special tree with $m$ edges, where $m \geq 8$. If $r_{k} \leq$ $m-d(v)+\max \{\ell(v), 1\}$ for all $v \in V(T)$, and $m / k<4$, then $T$ has a 2-good r-exact edge-coloring.

Proof. Let $x$ be the special vertex of $T$. Note $\min _{v}\{m-d(v)+\max \{\ell(v), 1\}\}=$ $m-d(x)+\max \{\ell(x), 1\}$. Form $T^{\prime}$ by replacing each copy of $K_{1,3}$ in $T$ that has $x$ as a leaf with a copy of $P_{4}$ having $x$ as a leaf. Lemma 3.5 will apply to give an edge coloring of $T^{\prime}$. For the copies of $K_{1,3}$ replaced with paths, assign the edge incident to $x$ the same color as in $T^{\prime}$, and assign the other edges the remaining colors. The resulting edge-coloring is 2 -good and $r$-exact. To apply Lemma 3.5, it suffices to show that the inequalities in the hypothesis of Lemma 3.5 are satisfied for $T^{\prime}$.

For $T^{\prime}$, we have $r_{k} \leq m-d(v)+\max \{\ell(v), 1\}$ for all $v$ if and only if the inequality holds for $v=x$. Note that $T^{\prime}$ also has $m$ edges, and $d_{T}(x)=d_{T^{\prime}}(x)$ and $\ell_{T}(x)=\ell_{T^{\prime}}(x)$. Given $d_{1}, d_{2}$, and $d_{3}$ defined as in Lemma 3.5 for $T^{\prime}$, let $j=c_{r}\left(d_{2}+d_{3}\right)$. Note that $d_{1}=\ell(x)$. In the case $c_{r}\left(d_{3}\right)<j$, since $r_{k} \leq$ $m-d(x)+\max \{\ell(x), 1\}$ is given, it follows that $r_{i} \leq m-d(x)+\max \{\ell(x), 1\}$ for all $i$. Hence Lemma 3.5 applies unless $r_{j}=m-d(x)+1>m-d(x)+\ell(x)$, which requires $\ell(x)=d_{1}=0$. If $j<k$, then $2 d_{2}+3 d_{3}=m \geq r_{j}+r_{k}=$ $2(m-d(x)+2)=2 d_{2}+4 d_{3}+2$, a contradiction. If $j=k$, then using color $k$ on the edges not incident to $x$ and using the other colors on the other edges 
yields a 2-good $r$-exact edge-coloring of $T^{\prime}$. Hence we need only consider the case $c_{r}\left(d_{3}\right)=j$.

Let $L=\sum_{i<j} r_{i}$. Note that $L<d_{3}$, since $c_{r}\left(d_{3}\right)=j$; we need $r_{j} \leq d(x)+$ $L$ to apply Lemma 3.5. Suppose $r_{j} \geq d(x)+L+1$. Since $r$ is nondecreasing, $r_{i} \geq r_{j}$ for $i \geq j$, and hence

(1) $m=\sum_{i} r_{i} \geq L+\sum_{i \geq j}(d(x)+L+1) \geq L+(k-j+1)(d(x)+L+1)$.

If $j \leq k-2$, then $m \geq L+3(d(x)+L+1)>3 d(x)$, a contradiction since $m=3 d_{3}+2 d_{2}+d_{1} \leq 3\left(d_{3}+d_{2}+d_{1}\right)=3 d(x)$. If $j=k$, then since $L<d_{3}$ and $r_{j} \leq m-d(x)+\ell(x)$, we have

$$
m=L+r_{k}<d_{3}+m-d(x)+\ell(x)=3 d_{3}+d_{2}+d_{1} \leq m,
$$

a contradiction. Therefore, $j=k-1$.

Substituting $k-j=1$ into (1) yields $m \geq 2 d(x)+3 L+2$. Using also $m \leq 3 d(x)$ obtains $d(x) \geq 3 L+2$, and hence $m \geq 9 L+6$. On the other hand, since $j \leq L+1$ and $m<4 k$, we have $m<4 k=4(j+1) \leq 4 L+8$. Thus $9 L+6 \leq m<4 L+8$, which implies $L=0$, and so $6 \leq m<8$, a contradiction since $m \geq 8$.

Applying Corollary 1.4, we have the following corollary of Theorem 4.2.

Corollary 3.8. Let $T$ be a special tree with $m$ edges, where $m>8$. Let $G$ be the cartesian product of $G_{1}, \ldots, G_{k}$, where each $G_{i}$ is either an $r_{i}$ regular bipartite graph or a $2 r_{i}$-regular graph. If $r_{k} \leq m-d(v)+\ell(v)$ for all $v \in V(T)$, and $m / k<4$, then $G$ has a $T$-decomposition.

\section{Weakly 2-good edge-coloring of general trees}

As mentioned, Corollary 3.8 fails for general trees, since the conditions $r_{k} \leq$ $m-d(v)+\ell(v)$ and $m / k<4$ are not sufficient for paths of length at least 6 to have a 2-good $r$-exact edge-coloring. However, existence of a 2-good $r$-exact edge-coloring in $T$ is not a necessary condition for $G$ to have a $T$ decomposition, so there should be a condition weaker than this that still suffices for $G$ to have a $T$-decomposition.

Definition 4.1. A 3-bounded edge-colored path in $T$ is weakly 2-bounded if either it is 2-bounded or it has a color appearing only on a 3-edge subpath whose two internal vertices have degree 2 in $T$. An edge-coloring of $T$ is weakly 2 -good if every path is weakly 2 -bounded. 
Theorem 4.2. Let $T$ be a special tree with $m$ edges. If $r_{k} \leq m-d(v)+$ $\max \{\ell(v), 1\}$ for all $v \in V(T)$, and $m / k<4$, then $T$ has a weakly 2-good r-exact edge-coloring.

Proof. Note that $T$ has a 2-good $r$-exact edge-coloring by Lemma 3.7 when $m \geq 8$. When $m \leq 7$, assign colors $1, \ldots, k$ in order to the edges in the increasing order of the distance from the special vertex. This yields a weakly 2-good edge-coloring of $T$.

We use a result of Kouider and Lonc [6] to show in Theorem 4.4 that the existence of a weakly 2-good $r$-exact edge-coloring of $T$ guarantees a $T$-decomposition in $G$. We will show later that such an edge-coloring exists in any tree with $m$ edges, including a path, if $r_{k} \leq\left\lceil\frac{m+1}{2}\right\rceil$ and $m / k<4$.

Theorem 4.3 (Kouider and Lonc [6]). Each $2 m$-regular graph $G$ with girth at least $(m+3) / 2$ has a $P_{m+1}$-decomposition with the property that each vertex of $G$ occurs as an endpoint in exactly two of the copies of $P_{m+1}$.

Theorem 4.4. Let $r$ be a list of positive integers with sum $m$. Let $T$ be a tree with $m$ edges, and let $G$ be the cartesian product of graphs $G_{1}, \ldots, G_{k}$, where $G_{i}$ is $2 r_{i}$-regular, for all $i$. If $T$ has a weakly 2-good $r$-exact edge-coloring, then $G$ has a $T$-decomposition.

Proof. For each $i$, let $\mathbf{F}_{\mathbf{i}}$ be a 2 -factorization of $G_{i}$. Consider a bijection that pairs each edge of color $i$ in $T$ with a 2-factor in $\mathbf{F}_{\mathbf{i}}$. In a weakly 2-good edgecoloring $f$ of $T$, the internal vertices of each monochromatic 3-edge path in $T$ have degree 2 in $T$. Let $T^{\prime}$ be the tree obtained from $T$ by shrinking each monochromatic 3-edge path to an edge having the same endpoints and the same color. Let $E^{\prime}\left(T^{\prime}\right)$ be the set of edges in $T^{\prime}$ that arise by shrinking monochromatic 3-edge paths. Let $f^{\prime}$ be the edge-coloring of $T^{\prime}$ that arises from $f$ by shrinking these paths. We claim that $f^{\prime}$ is 2 -good.

Each path $P$ in $T^{\prime}$ corresponds to a path $Q$ in $T$. Since $f$ is weakly 2-good, $Q$ is either 2-bounded or has a color appearing only on a 3-edge subpath whose internal vertices have degree 2 in $T$. Since every monochromatic $3-$ edge path in $T$ is shrunk to an edge in $T^{\prime}$, the corresponding path $P$ in $T^{\prime}$ is 2-bounded. Hence $f^{\prime}$ is 2-good.

The edges of a monochromatic 3-edge path in $T$ of color $i$ correspond to three 2-factors in $\mathbf{F}_{\mathbf{i}}$ that together form a 6-regular subgraph $H$ in $G_{i}$. Consider the $P_{4}$-decomposition guaranteed by Theorem 4.3 (note that $H$ always has girth at least $(m+3) / 2$ when $m=3)$. For each copy of $P_{4}$ in the decomposition of $H$, delete the edges and add an edge joining the endpoints of the copy. By the property that each vertex of $H$ occurs as an endpoint exactly twice in the decomposition, the resulting object $H^{\prime}$ is a 2-regular 
loopless multigraph. Obtain $G_{i}^{\prime}$ from $G_{i}$ by replacing $H$ with the resulting $H^{\prime}$ for each 3-edge path with color $i$ in $f$. After doing this for all $i$, let $G^{\prime}$ be the cartesian product of $G_{1}^{\prime}, \ldots, G_{k}^{\prime}$.

Since $T^{\prime}$ has a 2-good edge-coloring, $G^{\prime}$ has a $T^{\prime}$-decomposition, by Theorem 2.1. We extend each copy of $T^{\prime}$ to a copy of $T$, yielding a $T$ decomposition of $G$. For each $e \in E^{\prime}\left(T^{\prime}\right)$, replace the edge in each copy of $T^{\prime}$ that represents $e$ with a 3 -edge path having the same endpoints, yielding a copy of $T$, since this is the reverse of how $T^{\prime}$ was obtained from $T$.

Each edge $e$ in $E^{\prime}\left(T^{\prime}\right)$ corresponds to a 2-factor forming a copy of $H^{\prime}$ in $G^{\prime}$ whose edges appear as $e$ in distinct copies of $T^{\prime}$. This copy of $H^{\prime}$ arose from a copy of $H$ in $G$ with each edge in the copy of $H^{\prime}$ corresponding to a 3-edge path in the copy of $H$. Thus the 3-edge paths in all copies of $T$ that represent the 3 -edge path corresponding to $e$ decompose the copies of $H$, and the copies of $T$ form a $T$-decomposition of $G$.

By Lemma 2.5 and Example 2.6, the condition $m / k<4$ suffices for paths to have $r$-exact edge-colorings that are 3 -good, but not 2 -good. However, the condition $m / k<4$ does suffice for a weakly 2-good $r$-exact edge-coloring.

Lemma 4.5. If $m / k<4$, then $P_{m+1}$ has a weakly 2-good $r$-exact edgecoloring.

Proof. We use induction on $k$. If $k=1$, then $m \leq 3$, and giving all edges the same color is weakly 2-good. Consider $k>1$. Always $r_{1} \leq 3$. If $m<$ $r_{1}+4(k-1)$, then split $P$ into a subpath $P^{\prime}$ with $m-r_{1}$ edges and a subpath $P^{\prime \prime}$ with $r_{1}$ edges. Assign color 1 to the $r_{1}$ edges of $P^{\prime \prime}$. Since $k-1$ colors remain for $P^{\prime}$, which has fewer than $4(k-1)$ edges, by the induction hypothesis $P^{\prime}$ has a weakly 2-good $r^{\prime}$-exact edge-coloring. Since $P^{\prime}$ and $P^{\prime \prime}$ use disjoint sets of colors, the full edge-coloring is weakly 2 -good.

If $m \geq r_{1}+4(k-1)$, then $r_{k} \geq \frac{m-r_{1}}{k-1} \geq 4$. Split $P_{m+1}$ into a subpath $P^{\prime}$ with $m-4$ edges and a subpath $P^{\prime \prime}$ of length 4 . Let $r^{\prime}$ be the list $r_{2}, \ldots, r_{k}-$ $\left(4-r_{1}\right)$. Since $(m-4) /(k-1)<4$, the induction hypothesis implies that $P^{\prime}$ has a weakly 2 -good $r^{\prime}$-exact edge-coloring. For the remaining four edges, assign $r_{1}$ edges color 1 and $4-r_{1}$ edges color $k$, with color $k$ not being assigned to the edge incident to $P^{\prime}$. The full edge-coloring is weakly 2 -good, since $r_{1} \leq 3$.

In the proof of Lemma 4.5 , we split $P_{m+1}$ into two paths colored using an appropriate "split" of $r$ into two lists. The next lemma discusses such numerical splits in more generality and helps in showing that $r_{k} \leq\left\lceil\frac{m+1}{2}\right\rceil$ and $m / k<4$ together are sufficient for any tree to have a weakly 2 -good $r$ exact edge-coloring. The essential mean of a list is the average of its nonzero 
terms. A list with sum $m$ is half-bounded if every term is at most $\left\lceil\frac{m+1}{2}\right\rceil$, and it is nearly half-bounded if every term is at most $\left\lfloor\frac{m+3}{2}\right\rfloor$. A split of a nonnegative $k$-tuple $r$ consists of two nonnegative $k$-tuples $r^{\prime}$ and $r^{\prime \prime}$ such that $r_{i}^{\prime}+r_{i}^{\prime \prime}=r_{i}$ for $1 \leq i \leq k$. The two lemmas below will be used to prove our main theorem. The proof of Lemma 4.6 is somewhat technical, so we postpone it to Section 5 .

Lemma 4.6. Let $r$ be a nearly half-bounded list with sum $m$.

a) If $0<m^{\prime}<m$, then $r$ splits into half-bounded lists $r^{\prime}$ and $r^{\prime \prime}$ having essential means at most $m / k$ and sums $m^{\prime}$ and $m-m^{\prime}$, respectively.

b) Let $b=m-k\left\lfloor\frac{m}{k}\right\rfloor$. If $3 k \leq m<\left\lfloor\frac{m}{k}\right\rfloor(k+1)$, then for $m^{\prime}$ with $b<m^{\prime}<m-b$ the essential means can be required to be less than $\lfloor m / k\rfloor$.

Let a nontrivial star be a star with at least one edge, and let a penultimate edge in a tree be an edge whose deletion leaves a component that is a nontrivial star.

Lemma 4.7. Let $T$ be a tree with $m$ edges.

a) If $T$ is not a special tree, then $T$ has an edge $e$ whose deletion leaves components $T^{\prime}$ and $T^{\prime \prime}$ such that $T^{\prime}$ is a special tree with at least three edges whose vertex incident to e can designated as the special vertex.

b) If $T$ is neither a path nor a star, then $T$ has an edge $e$ whose deletion leaves components $T^{\prime}$ and $T^{\prime \prime}$ such that $T^{\prime}$ is a nontrivial star and $T^{\prime \prime}+e$ is not a path.

Proof. For a longest path in $T$, let $(1, b, c)$ be the degrees of the first three vertices. Choose $P$ to be a longest path that lexicographically maximizes $(1, b, c)$. Let $z, y, x, w$ be the first four vertices of $P$ in order ( $T$ is not a star).

(a) If $d_{T}(y) \geq 4$, then since the component of $T-x y$ containing $z$ is a star (and hence a special tree) with at least three edges, the edge $x y$ suffices. If $d_{T}(y)=3$, then by the choice of $P$, all neighbors of $x$ other than $w$ have degree at most 3 in $T$. The component of $T-w x$ containing $z$ is a special tree, and hence $w x$ suffices. Since $d_{T}(y) \geq 2$, we may henceforth assume $d_{T}(y)=2$.

If $d_{T}(x) \geq 3$, then by the choice of $P$ every neighbor of $x$ other than $y$ has degree at most 2 , since $d_{T}(y)=2$. The component of $T-w x$ containing $z$ is a special tree with at least three edges, and again $w x$ suffices. The remaining case is $d_{T}(y)=d_{T}(x)=2$. By the choice of $P$, every component of $T-w$ except one is isomorphic to a path of length 2 . Since $T$ is not a special tree, $w$ has a neighbor $v$ on $P$ other than $x$. Thus the component of $T-v w$ containing $z$ is a special tree with at least three edges, and hence $v w$ suffices. 
(b) The edge $x y$ suffices unless the component of $T-x y$ not containing $z$ is a path $P^{\prime}$ starting with $x$. Since $T$ is not a star, $P^{\prime}$ has length at least 1. Let $e$ be the edge of $T$ incident to the last edge of $P^{\prime}$. The component of $T-e$ not containing $z$ is $P_{2}$, a nontrivial star. Since $T$ is not a path, the component of $T-x y$ containing $z$ is a star with at least two edges, and adding $e$ completes a subgraph that is not a path.

Our main result in this section gives numerical conditions on $r$ to imply that every cartesian product of regular graphs with degrees $2 r_{1}, \ldots, 2 r_{k}$ has a $T$-decomposition when $T$ is any tree with $\sum r_{i}$ edges.

Theorem 4.8. Let $T$ be a tree with $m$ edges. If $r_{k} \leq\left\lceil\frac{m+1}{2}\right\rceil$ and $m / k<4$, then $T$ has a weakly 2-good r-exact edge-coloring.

Proof. We use induction on $m$. If $m \leq 7$, then $T$ is either a special tree or a path. Consider $m \geq 8$, and thus $k \geq 3$. If $T$ is a special tree or a path, then $T$ has a weakly 2-good edge-coloring, by Lemma 4.5 and Theorem 4.2. Thus we may assume that $T$ is neither a special tree nor a path. Since at most one term in $r$ equals $\left\lceil\frac{m+1}{2}\right\rceil$, if there are two largest terms, then they are less than $\left\lceil\frac{m+1}{2}\right\rceil$. Since $m / k<4$, we have $r_{1} \in\{1,2,3\}$.

Case 1: $r_{1}=1$. Since the list $\left(r_{2}, \ldots, r_{k}\right)$ has sum $m-1$, and $r_{k} \leq$ $\left\lceil\frac{m+1}{2}\right\rceil=\left\lfloor\frac{(m-1)+3}{2}\right\rfloor$, the list is nearly half-bounded. Since $T$ is not a special tree and $m \geq 8$, Lemma 4.7a yields an edge $e$ whose deletion leaves components $T^{\prime}$ and $T^{\prime \prime}$ such that both components have at least three edges. (If $T^{\prime \prime}$ does not have three edges, then it and $e$ can be added to $T^{\prime}$, making $T$ a special tree).

If $4 k-3 \leq m \leq 4 k-1$, then $4(k-1) \leq m-1 \leq 4(k-1)+2$. It follows that $(m-1)-(k-1)\left|\frac{m-1}{k-1}\right| \leq 2$, and hence Lemma 4.6b applies, since $\left|E\left(T^{\prime}\right)\right|>2$ and $\left|E\left(T^{\prime \prime}\right)\right|>2$. Hence the list $r_{2}, \ldots, r_{k}$ splits into half-bounded lists $r^{\prime}$ and $r^{\prime \prime}$ with sums $\left|E\left(T^{\prime}\right)\right|$ and $\left|E\left(T^{\prime \prime}\right)\right|$, respectively, and both of their essential means are less than 4 . If $m \leq 4 k-4$, then $m-1 \leq 4(k-1)-1$, and hence by Lemma 4.6 a the list $r_{2}, \ldots, r_{k}$ splits into half-bounded lists $r^{\prime}$ and $r^{\prime \prime}$ with sums $\left|E\left(T^{\prime}\right)\right|$ and $\left|E\left(T^{\prime \prime}\right)\right|$, respectively, and both of their essential means are less than 4.

Therefore, in either case, the list $r_{2}, \ldots, r_{k}$ splits into half-bounded lists $r^{\prime}$ and $r^{\prime \prime}$ with sums $\left|E\left(T^{\prime}\right)\right|$ and $\left|E\left(T^{\prime \prime}\right)\right|$ such that both essential means are less than 4 . Assign color 1 to the edge $e$, and apply the induction hypothesis to both $T^{\prime}$ and $T^{\prime \prime}$ to obtain weakly 2 -good edge-colorings. The full edgecoloring is weakly 2 -good.

Case 2: $r_{1}=2$. Here the list $\left(r_{1}-1, r_{2}, \ldots, r_{k}\right)$ is nearly half-bounded and has sum $m-1$, which is at most $4 k-2$. Since $m \geq 8$, there is an edge $e$ of 
$T$ whose deletion leaves nontrivial components $T^{\prime}$ and $T^{\prime \prime}$. By Lemma 4.6a, the list $r_{1}-1, r_{2}, \ldots, r_{k}$ splits into half-bounded lists $r^{\prime}$ and $r^{\prime \prime}$ with sums $\left|E\left(T^{\prime}\right)\right|$ and $\left|E\left(T^{\prime \prime}\right)\right|$ whose essential means are less than 4 . Assign color 1 to $e$, and apply the induction hypothesis to both $T^{\prime}$ and $T^{\prime \prime}$ to obtain weakly 2 -good edge-colorings. The full edge-coloring is weakly 2 -good.

Case 3: $r_{1}=3$. Consider the list $\left(r_{2}, \ldots, r_{k}\right)$. Since $m-3 k \leq k-1$, we have $m-3 k \leq \frac{m-3 k}{2}+\frac{k-1}{2}=\frac{m+1}{2}-k-1$. Also, since $r_{i} \geq r_{1}=3$, we have $r_{k}=m-\sum_{i=1}^{k-1} r_{i} \leq m-3(k-1)$. Hence $r_{k} \leq m-3 k+3 \leq \frac{m+1}{2}-k+2 \leq$ $\left\lfloor\frac{(m-3)+3}{2}\right\rfloor$, since $k \geq 3$. Therefore, $\left(r_{2}, \ldots, r_{k}\right)$ is nearly half-bounded.

Since $T$ is not a path or star, by Lemma $4.7 \mathrm{~b}$ it has an edge $e$ whose deletion leaves components $T^{\prime}$ and $T^{\prime \prime}$ such that $T^{\prime \prime}+e$ is not a path. Let $e_{1}$ and $e_{2}$ be pendant edges of $T^{\prime \prime}+e$ other than $e$. Since $r_{2}, \ldots, r_{k}$ is nearly half-bounded and has sum $m-3$, which is at most $4(k-1)$, by Lemma $4.6 \mathrm{a}$ it splits into half-bounded lists $r^{\prime}$ and $r^{\prime \prime}$ with sums $\left|E\left(T^{\prime}\right)\right|-2$ and $\left|E\left(T^{\prime \prime}\right)\right|$ whose essential means are less than 4 . Assign color 1 to all of $\left\{e, e_{1}, e_{2}\right\}$, and apply the induction hypothesis to the two remaining trees to obtain weakly 2 -good edge-colorings. The full edge-coloring is weakly 2 -good.

\section{List splittability}

In this section, we prove Lemma 4.6 (as Lemma 5.3). Recall that a split of the $k$-tuple $r$ consists of two nonnegative $k$-tuples $r^{\prime}$ and $r^{\prime \prime}$ such that $r_{i}^{\prime}+r_{i}^{\prime \prime}=r_{i}$ for $1 \leq i \leq k$. Given $m^{\prime}$ with $0<m^{\prime}<m=\sum r_{i}$, let $m^{\prime \prime}=m-m^{\prime}$. We will first give sufficient conditions for a split of $r$ into half-bounded lists $r^{\prime}$ and $r^{\prime \prime}$ with sums $m^{\prime}$ and $m^{\prime \prime}$, respectively, such that both $r^{\prime}$ and $r^{\prime \prime}$ have at least certain numbers of nonzero terms. We apply this in Lemma 5.3 to show that if $r$ is nearly half-bounded (meaning $r_{k} \leq\left\lfloor\frac{m+3}{2}\right\rfloor$ ), then $r$ splits into halfbounded lists $r^{\prime}$ and $r^{\prime \prime}$ having essential means at most the essential mean $m / k$ of $r$. Under additional hypotheses, for most values of $m^{\prime}$ the essential means of $r^{\prime}$ and $r^{\prime \prime}$ can also be required to be less than $\lfloor m / k\rfloor$. We state the first lemma using $x$ rather than $m^{\prime}$ because we will also apply it in the complementary situation where $x=m^{\prime \prime}$.

Lemma 5.1. Let $r$ be a nearly half-bounded list with sum $m$. Fix integers $x$ and $y$ with $0<x<m$ and $0 \leq y<k$. Let $t_{i}=\min \left\{r_{i},\left\lceil\frac{m-x+1}{2}\right\rceil\right\}$ for $1 \leq i \leq k$. Let $j=\max \left\{i: r_{i} \leq\left\lceil\frac{m-x+1}{2}\right\rceil\right\}$. Let $S$ be a subset of $\{1, \ldots, j\}$ with size $\max \{0,(y+1)-(k-j)\}$. Let $s_{i}=r_{i}-t_{i}+1$ for $i \in S$ and $s_{i}=r_{i}-t_{i}$ for $i \notin S$. If (1) $y+1 \leq x$ and (2) either $2 y+1 \leq x$ or $2(k-y) \geq m-x$, then $\sum s_{i} \leq x$. Furthermore, $s$ has at least $y+1$ nonzero terms. 
Proof. Note that $r_{i}-t_{i}=\max \left\{0, r_{i}-\left\lceil\frac{m-x+1}{2}\right\rceil\right\}$; in particular $r_{i}-t_{i}=0$ and $s_{i}=1$ for $i \in S$. By the choice of $j$,

$$
\sum_{i=1}^{k} s_{i}=|S|+\sum_{i>j}\left(r_{i}-\left\lceil\frac{m-x+1}{2}\right\rceil\right) .
$$

If $j=k$, then $\sum_{i=1}^{k} s_{i}=|S|=y+1 \leq x$. If $j \leq k-2$, then $\sum_{i>j}\left\lceil\frac{m-x+1}{2}\right\rceil \geq$ $2\left\lceil\frac{m-x+1}{2}\right\rceil$. Since $|S| \leq j$ and $\sum_{i>j} r_{i} \leq m-j$,

$$
\sum_{i=1}^{k} s_{i} \leq j+m-j-(m-x+1)<x .
$$

If $j=k-1$, then $\sum_{i=1}^{k} s_{i}=y+r_{k}-\left\lceil\frac{m-x+1}{2}\right\rceil$. Since $r_{k} \leq\left\lfloor\frac{m+3}{2}\right\rfloor$, in the case $2 y+1 \leq x$ we have

$$
\sum_{i=1}^{k} s_{i} \leq\left\lfloor\frac{x-1}{2}\right\rfloor+\left\lfloor\frac{m+3}{2}\right\rfloor-\left\lceil\frac{m-x+1}{2}\right\rceil \leq x .
$$

Since also $r_{k} \leq m-k+1$, in the case $2(k-y) \geq m-x$ we have

$$
\begin{aligned}
\sum_{i=1}^{k} s_{i} & \leq y+m-k+1-\left\lceil\frac{m-x+1}{2}\right\rceil \\
& =y-k+1+\left\lfloor\frac{m-x-1}{2}\right\rfloor+x \\
& \leq y-k+1+k-y-1+x=x .
\end{aligned}
$$

To count the nonzero terms in $s$, note that if $i>j$, then $s_{i}>0$. If $i \leq j$, then $s_{i}>0$ for $i \in S$. Hence $s$ has at least $|S|+k-j$ nonzero terms, which is at least $y+1$.

When comparing lists of the same length, an expression like $r^{\prime} \leq t^{\prime}$ means $r_{i}^{\prime} \leq t_{i}^{\prime}$ for all $i$. Our plan is as follows.

Remark 5.2. We will first define $t^{\prime}$ and $t^{\prime \prime}$ as instances of the list $t$ in Lemma 5.1 with $x=m-m^{\prime}$ and $x=m^{\prime}$, respectively. By the definition of $t$, any list $r^{\prime}$ with $r^{\prime} \leq t^{\prime}$ and $\sum r_{i}^{\prime}=m^{\prime}$ is half-bounded; similarly for $r^{\prime \prime}$.

We will next obtain lists $s^{\prime}$ and $s^{\prime \prime}$ as instances of the list $s$ in Lemma 5.1 such that $s^{\prime} \leq r-s^{\prime \prime}$ and $\sum s_{i}^{\prime} \leq m^{\prime} \leq \sum\left(r_{i}-s_{i}^{\prime \prime}\right)$. Given such lists, we produce $r^{\prime}$ by starting with $s^{\prime}$ and augmenting elements of the list, while 
keeping the $i$ th element at most $r_{i}-s_{i}^{\prime \prime}$, until we reach sum $m^{\prime}$. Since $r^{\prime} \geq$ $s^{\prime}$, the list $r^{\prime}$ has at least as many nonzero terms as $s^{\prime}$. Similarly, since $r-r^{\prime \prime}=r^{\prime} \leq r-s^{\prime \prime}$ implies $r^{\prime \prime} \geq s^{\prime \prime}$, the list $r^{\prime \prime}$ has at least as many nonzero terms as $s^{\prime \prime}$. Furthermore, $s^{\prime}$ and $s^{\prime \prime}$ will be defined so that $r-s^{\prime \prime} \leq t^{\prime}$ and $r-s^{\prime} \leq t^{\prime \prime}$. It then follows that $r^{\prime} \leq r-s^{\prime \prime} \leq t^{\prime}$ and $r^{\prime \prime}=r-r^{\prime} \leq r-s^{\prime} \leq t^{\prime \prime}$, and hence $r^{\prime}$ and $r^{\prime \prime}$ are half-bounded.

It remains to obtain such lists $s^{\prime}$ and $s^{\prime \prime}$ having sufficiently many nonzero terms (to make the essential means small). We will do this using special sets $S^{\prime}$ and $S^{\prime \prime}$ in the manner in which $s$ is defined from $t$ in Lemma 5.1. We will need to ensure that the specifications of $S^{\prime}$ and $S^{\prime \prime}$ do not prevent $s^{\prime} \leq r-s^{\prime \prime}$.

Lemma 5.3. Let $r$ be a nearly half-bounded list with sum $m$.

a) If $0<m^{\prime}<m$, then $r$ splits into half-bounded lists $r^{\prime}$ and $r^{\prime \prime}$ with sums $m^{\prime}$ and $m^{\prime \prime}$ having essential means at most $m / k$.

b) Let $b=m-k\left\lfloor\frac{m}{k}\right\rfloor$. If $3 k \leq m \leq\left\lfloor\frac{m}{k}\right\rfloor(k+1)$ and $b<m^{\prime}<m-b$, then the essential means of $r^{\prime}$ and $r^{\prime \prime}$ can be required to be less than $\lfloor m / k\rfloor$.

Proof. We will define parameters $k^{\prime}$ and $k^{\prime \prime}$ and construct lists $s^{\prime}$ and $s^{\prime \prime}$ with $k^{\prime}+1$ nonzero terms and $k^{\prime \prime}+1$ nonzero terms, respectively, such that $s^{\prime} \leq r-s^{\prime \prime}$ and $\sum s_{i}^{\prime} \leq m^{\prime} \leq \sum\left(r_{i}-s_{i}^{\prime \prime}\right)$. The lists $s^{\prime}$ and $s^{\prime \prime}$ will be instances of $s$ obtained from $r$ as in Lemma 5.1, using parameters $x$ and $y$ and an appropriate set $S$. For $s^{\prime}$, we use $x=m^{\prime}$ and $y=k^{\prime}$. For $s^{\prime \prime}$, we use $x=m-m^{\prime}$ and $y=k^{\prime \prime}$. We let $S^{\prime}$ and $S^{\prime \prime}$ denote the sets to be used as $S$ in determining $s^{\prime}$ and $s^{\prime \prime}$, respectively. Similarly, let $j^{\prime}$ and $j^{\prime \prime}$ denote the index $j$ computed in the two instances. Let $k^{\prime}=\left\lfloor\frac{m^{\prime}}{a}\right\rfloor$ and $k^{\prime \prime}=k-k^{\prime}-\delta$, where $a$ and $\delta$ will be defined differently for part (a) and part (b). In both cases, $k^{\prime} \leq \frac{m^{\prime}}{a}<k^{\prime}+1$.

(a) Since the conclusion is obvious if $r_{i}=1$ for all $i$, we assume $r_{k} \geq 2$. We set $a=m / k$ and $\delta=1$, so $k^{\prime \prime}=k-k^{\prime}-1$. To see that having lists $s^{\prime}$ and $s^{\prime \prime}$ as described above suffices, note that any list $r^{\prime}$ with $r^{\prime} \geq s^{\prime}$ and sum $m^{\prime}$ has at least $k^{\prime}+1$ nonzero terms, and hence has essential mean at most $\frac{m^{\prime}}{k^{\prime}+1}$, which is less than $a$ by the choice of $k^{\prime}$. Similarly, any list $r^{\prime \prime}$ with $r^{\prime \prime} \geq s^{\prime \prime}$ and sum $m-m^{\prime}$ has essential mean at most $\frac{m-m^{\prime}}{k-k^{\prime}}$, which is at most $a$ since $m k^{\prime} \leq m^{\prime} k$. As noted in Remark 5.2, $s^{\prime} \leq r-s^{\prime \prime}$ and $\sum s_{i}^{\prime} \leq m^{\prime} \leq \sum\left(r_{i}-s_{i}^{\prime \prime}\right)$ allows us to obtain such $r^{\prime}$ and $r^{\prime \prime}$ by iteratively augmenting terms.

To apply Lemma 5.1, we need to define $S^{\prime}$ and $S^{\prime \prime}$ appropriately. Each choice of $y\left(k^{\prime}\right.$ or $k^{\prime \prime}$, respectively) must be less than $k$. We have $k^{\prime}=$ $\left\lfloor\frac{m^{\prime} k}{m}\right\rfloor<k$ and $k^{\prime \prime}=k-k^{\prime}-1<k$. Since we want $S^{\prime}$ to be a set of size $\max \left\{0,\left(k^{\prime}+1\right)-\left(k-j^{\prime}\right)\right\}$, let $S^{\prime}=\left\{k-k^{\prime}, \ldots, j^{\prime}\right\}$; this set is empty if $k^{\prime}+1 \leq k-j^{\prime}$. Similarly, since $\left(k^{\prime \prime}+1\right)-\left(k-j^{\prime \prime}\right)=j^{\prime \prime}-k^{\prime}$ when $y=k^{\prime \prime}=$ $k-k^{\prime}-1$, we need $\left|S^{\prime \prime}\right|=\max \left\{0, j^{\prime \prime}-k^{\prime}\right\}$. We set $S^{\prime \prime}=\left\{1, \ldots, j^{\prime \prime}-k^{\prime}\right\}$, 
except $S^{\prime \prime}=\left\{1, \ldots, j^{\prime \prime}-k^{\prime}-1\right\} \cup\left\{j^{\prime \prime}\right\}$ when $j^{\prime \prime}=k$. As in Lemma 5.1, $s_{i}^{\prime}=1$ for $i \in S^{\prime}$ and $s_{i}^{\prime}=\max \left\{0, r_{i}-\left\lceil\frac{m-m^{\prime}+1}{2}\right\rceil\right\}$ for $i \notin S^{\prime}$. Similarly, $r_{i}-s_{i}^{\prime \prime}=r_{i}-1$ for $i \in S^{\prime \prime}$ and $r_{i}-s_{i}^{\prime \prime}=\min \left\{r_{i},\left\lceil\frac{m^{\prime}+1}{2}\right\rceil\right\} \geq 1$ for $i \notin S^{\prime \prime}$.

We need to show $s^{\prime} \leq r-s^{\prime \prime}$. Since $r_{i} \leq r_{k} \leq\left\lfloor\frac{m+3}{2}\right\rfloor \leq\left\lceil\frac{m-m^{\prime}+1}{2}\right\rceil+$ $\left\lceil\frac{m^{\prime}+1}{2}\right\rceil$, we have $s_{i}^{\prime} \leq r_{i}-s_{i}^{\prime \prime}$ when $i \notin S^{\prime}$ and $i \notin S^{\prime \prime}$. The cases when $i$ is in just one of $S^{\prime}$ and $S^{\prime \prime}$ are immediate. When $i \in S^{\prime} \cap S^{\prime \prime}$, we need $r_{i} \geq 2$. We have $i \in S^{\prime} \cap S^{\prime \prime}$ only when $j^{\prime \prime}=k$ and $i=k$; now $r_{k} \geq 2$ suffices.

To show $\sum s_{i}^{\prime} \leq m^{\prime} \leq \sum\left(r_{i}-s_{i}^{\prime \prime}\right)$, we apply Lemma 5.1 twice. First consider $\sum s_{i}^{\prime} \leq m^{\prime}$. Recall that $a=m / k$. Note that $a>1$, since $r_{k} \geq 2$. Now $y=k^{\prime} \leq m^{\prime} / a<m^{\prime}=x$. When $a>2$, we have $2 y=2 k^{\prime} \leq 2 m^{\prime} / a<m^{\prime}=x$. When $a \leq 2$, since $\frac{m-m^{\prime}}{k-k^{\prime}} \leq a \leq 2$, we have $2(k-y)=2\left(k-k^{\prime}\right) \geq m-m^{\prime}=$ $m-x$. Hence the hypotheses of Lemma 5.1 hold, and we conclude $\sum s_{i}^{\prime} \leq m^{\prime}$.

To prove $m^{\prime} \leq \sum\left(r_{i}-s_{i}^{\prime \prime}\right)$, we show $\sum s_{i}^{\prime \prime} \leq m-m^{\prime}$. In this application of Lemma 5.1, $y=k^{\prime \prime}$ and $x=m-m^{\prime}$. Since $k^{\prime} \geq m^{\prime} / a$ and $k \leq m$, we have $y+1=k-k^{\prime} \leq k\left(1-\frac{m^{\prime}}{m}\right)=\frac{k}{m}\left(m-m^{\prime}\right) \leq x$. When $m^{\prime} / k^{\prime} \geq 2$, we have $2 y+1<2\left(k-k^{\prime}\right) \leq \frac{m^{\prime}}{k^{\prime}}\left(k-k^{\prime}\right) \leq m-m^{\prime}=x$. When $m^{\prime} / k^{\prime}<2$, we have $2(k-y)>2 k^{\prime}>m^{\prime}=m-x$. Hence again the hypotheses of Lemma 5.1 hold, and we conclude $\sum s_{i}^{\prime \prime} \leq m-m^{\prime}$.

(b) We set $a=\left\lfloor\frac{m}{k}\right\rfloor$. As in part (a), any list $r^{\prime}$ with $r^{\prime} \geq s^{\prime}$ and sum $m^{\prime}$ has essential mean less than $a$. If $m-m^{\prime}<a\left(k-k^{\prime}\right)$, then $k-k^{\prime}$ nonzero terms are enough for $r^{\prime \prime}$ to have essential mean less than $a$. Otherwise $m-m^{\prime}<$ $a\left(k-k^{\prime}+1\right)$ (since $\left.m^{\prime} \geq a k^{\prime}\right)$, and then $k-k^{\prime}+1$ nonzero terms are enough. Hence we set $k^{\prime \prime}=k-k^{\prime}-\delta$, where $\delta=1$ if $m-m^{\prime}<a\left(k-k^{\prime}\right)$ and $\delta=0$ if $m-m^{\prime} \geq a\left(k-k^{\prime}\right)$. Again we need $y$ ( $k^{\prime}$ or $k^{\prime \prime}$, respectively) to be less than $k$. We have $k^{\prime}=\left\lfloor\frac{m}{a}\right\rfloor<\left\lfloor\frac{m-b}{a}\right\rfloor<k$ and $k^{\prime \prime}=k-k^{\prime}-\delta<k$.

We define $S^{\prime}$ and $S^{\prime \prime}$ as follows. As in part (a), let $S^{\prime}=\left\{k-k^{\prime}, \ldots, j^{\prime}\right\}$. For $S^{\prime \prime}$, we set $S^{\prime \prime}=\left\{1, \ldots, j^{\prime \prime}-k^{\prime}+1\right\}$, except $S^{\prime \prime}=\left\{1, \ldots, j^{\prime \prime}-k^{\prime}\right\} \cup\left\{j^{\prime \prime}\right\}$ when $j^{\prime \prime}=k-1$ and $S^{\prime \prime}=\left\{1, \ldots, j^{\prime \prime}-k^{\prime}-1\right\} \cup\left\{j^{\prime \prime}-1, j^{\prime \prime}\right\}$ when $j^{\prime \prime}=k$. Again, we need $r_{i} \geq 2$. We have $i \in S^{\prime} \cap S^{\prime \prime}$ only when $j^{\prime \prime} \geq k-1$, in which case $S^{\prime} \cap S^{\prime \prime} \subseteq\{k-1, k\}$. If $k=1$, then $r_{k}=m \geq 3 k=3$. If $k \geq 2$, then since $r_{k} \leq\left\lfloor\frac{m+3}{2}\right\rfloor$ and $m \geq 3 k$ we have

$$
r_{k-1} \geq m-r_{k}-(k-2) \geq\left\lceil\frac{m-3}{2}\right\rceil-(k-2) \geq 2 .
$$

Now we show $\sum s_{i}^{\prime} \leq m^{\prime}$ and $\sum s_{i}^{\prime \prime} \leq m-m^{\prime}$ by applying Lemma 5.1 twice. To confirm the hypotheses of Lemma 5.1, it suffices to show $2 y+1 \leq x$ when $(y, x)=\left(k^{\prime}, m^{\prime}\right)$ and when $(y, x)=\left(k^{\prime \prime}, m-m^{\prime}\right)$. Since $a \geq 3$, we have $2 k^{\prime}+1=2\left\lfloor\frac{m^{\prime}}{a}\right\rfloor+1 \leq m^{\prime}$. Hence we conclude $\sum s_{i}^{\prime} \leq m^{\prime}$. Now consider $\sum s_{i}^{\prime \prime} \leq m-m^{\prime}$. When $\delta=0$, we have $2 k^{\prime \prime}+1=2\left(k-k^{\prime}\right)+1$ and $m-m^{\prime} \geq$ 
$a\left(k-k^{\prime}\right)$. Since $a \geq 3$ and $k^{\prime}<k$, we have $2\left(k-k^{\prime}\right)+1 \leq a\left(k-k^{\prime}\right) \leq m-m^{\prime}$. When $\delta=1$, we have $2 k^{\prime \prime}+1=2\left(k-k^{\prime}\right)-1$ and $m-m^{\prime}>a\left(k-k^{\prime}-1\right)$. Note that $2\left(k-k^{\prime}\right)-1 \leq a\left(k-k^{\prime}-1\right)$ is equivalent to $0<(a-2)\left(k-k^{\prime}\right)$, which again holds since $a \geq 3$ and $k^{\prime}<k$.

Remark 5.4. In the proof of Lemma 5.3(a) the essential mean of $r^{\prime}$ is actually less than the essential mean $m / k$ of $r$.

In Lemma $5.3(\mathrm{~b})$, the condition $m \geq 3 k$ can be relaxed to $m \geq 2 k$, but then $r_{k-1} \geq 2$ needs to be required, since $r_{k-1}=1$ can happen when $a=2$. However, the proof needs more case analysis and we do not need this strengthening. Since no list has essential mean less than 1, the condition $2 k \leq m$ cannot be relaxed more. With $m \geq 2 k$, the condition $r_{k-1} \geq 2$ cannot be relaxed more, as shown by the list $(1, \ldots, 1, k+1)$, where $k$ is even. When $m^{\prime}=k$, the list has no split consisting of half-bounded lists with essential means less than 2 .

\section{References}

[1] S. V. Avgustinovich, On the partition of a set of edges of a graph into isomorphic trees, (Russian) Diskretn. Anal. Issled. Oper. Ser. 14 (1997), 3-5, 115. MR1658773

[2] J. F. Fink, On the decomposition of $n$-cubes into isomorphic trees, $J$. Graph Theory 14 (1990), 405-411. MR1067234

[3] R. Häggkvist, Decompositions of complete bipartite graphs, in Surveys in Combinatorics, 1989 (J. Siemons, ed.) London Math. Soc. Lect. Notes 141, Cambridge Univ. Press (1989), 115-147. MR1036754

[4] D. König, Über Graphen und ihre Anwendung auf Determinantentheorie und Mengenlehre, Math. Ann. 77 (1916), 453-465. MR1511872

[5] H. Snevily, Combinatorics of finite sets, PhD Thesis, University of Illinois, 1991. MR2687109

[6] M. Kouider and Z. Lonc, Path decompositions and perfect path double covers, Australas. J. Combin. 19 (1999), 261-274. MR1695816

[7] J. Petersen, Die Theorie der regulären Graphen, Acta Math. 15 (1891), 193-220. MR1554815

[8] G. Ringel, Problem 25, in Theory of Graphs and its Applications (Proc. Symp. Smolenice 1963), Czech. Acad. Sci. (1964), 162. MR0179778 
KYLE F. JAO,

UNIVERSITY OF ILLINOIS

URBANA, IL

USA

E-mail address: fjao2@illinois.edu

Alexandr V. Kostochka

UNIVERSITY OF ILLINOIS

URBANA, IL

USA

ZheJIANg NoRmal University

JiNHUA

China

E-mail address: kostochk@math.uiuc.edu

Douglas B. West

ZheJiang NoRmal University

JiNHUA

China

UNIVERSITY OF ILLINOIS

URBANA, IL

USA

E-mail address: west@math.uiuc.edu

Received August 28, 2012 